\title{
Introducing Path Computation Element (PCE) in Optical Grid Networking
}

\author{
F. Cugini (1), S. Xu (2), H. Harai (2), F. Paolucci (3), L. Valcarenghi (3), P. Castoldi (3) \\ 1: CNIT, Pisa, Italy, e-mail: filippo.cugini@cnit.it \\ 2: NICT, Koganei, Tokyo, Japan \\ 3: Scuola Superiore Sant'Anna, Pisa, Italy
}

\begin{abstract}
A PCE-based scheme is proposed to enhance grid resource selection with network information. The amount of established grid services is increased without significantly affecting the service set up time.

\section{Introduction}

Current grid-enabled services typically rely on computational resource information to select the grid resources to exploit. However, in case of bandwidthgreedy grid services, introducing a wavelength-routed network is also promising for providing dedicated high-speed connections (i.e., lightpaths) among grid resources. Further, the knowledge of network information has the potential to significantly increase the overall service performance [1]. The recent introduction of the Path Computation Element (PCE) represents an appealing solution to provide and exploit a detailed knowledge of network resources.

establish the required connections among the $g$ network nodes to which grid resources are connected. PCE is not utilized and a distributed set up is exploited: ingress nodes are independently configured with just the indications on the path destinations. Network resource information provided by the routing protocol allows to locally compute in ingress nodes the strict routes towards the destinations. The signalling protocol is then responsible for the connection set up. In case of transparent networks, the signalling protocol also identifies the specific wavelength to use by exploiting the currently defined GMPLS features.
\end{abstract}

In this study, we first evaluate the benefits in terms of network resource utilization provided by a grid networking scheme exploiting the PCE capability to perform concurrent and synchronized path computations. Then, we propose a novel scheme for grid resource selection. The scheme resorts to the currently defined PCE architecture to provide a feedback to the grid service on the expected network resource utilization. In this way, the grid service can first evaluate an extended set of grid resources to exploit and, among them, select those that minimize the overall network resource utilization. The proposed scheme is particularly suitable in case of grid services for e-Science applications (e.g., electronic Very-LongBaseline Interferometry (e-VLBI) [2] that require high bandwidth connections and high level of connectivity among multiple grid resources (e.g., full mesh of lightpaths) and expect significant time duration (e.g., tens of minutes or above). Moreover, the proposed solution does not require additional protocol extensions and allows keeping separate the control between grid and network resources, which can thus belong to different organizations.

\section{Grid networking without PCE}

Current grid services typically exploit a scheme, here referred to as $U g D$, which selects the most Unloaded $g$ grid resources and performs Distributed path computation. UgD scheme performs grid resource selection according to information available at the grid layer only, i.e., among the available $G$ grid resources, the set of $g$ most unloaded grid resources is selected. In case of equally unloaded grid resources, a random choice is performed. Then, the network service provider exploits the GMPLS protocol suite to

\section{Proposed PCE-based Grid networking}

A first enhanced scheme, here referred to as $U g P$, is introduced when PCE is exploited by the network service provider. UgP scheme selects the most Unloaded $g$ grid resources and exploits PCE for path computation. UgP scheme behaves as UgD to select the grid resources to exploit. However, in this case, the path computation relies on the PCE capability to perform multiple concurrent and synchronized path computations. The PCE has full visibility of network resources and provides all strict route indications. In case of transparent network, PCE provides also indications on the specific wavelength to use [3], thus avoiding conflicts during resource reservation.

A further enhanced PCE-based scheme, here referred to as $U g P k$, is proposed. UgPk scheme selects the most Unloaded $g$ grid resources and exploits PCE not only for concurrent path computation but also to provide a feedback on $k$ grid resources $(k>g)$. The UgPk scheme with $k=g$ is equivalent to the UgP scheme. In the UgPk scheme, the most unloaded $g$ grid resources are first considered. In case of equally unloaded grid resources, up to $k$ equally unloaded resources are identified. The PCE is then required to perform all the $p$ path computations (e.g., $p=g(g-1) / 2$ in case of full mesh) for every combination of the required $g$ nodes out of the identified $k$ resources (i.e., $\quad c=k ! /(g !(k-g) !)$ combinations). The $p$ path computations together with the computed metric values are then returned to the grid service which selects for service (i.e., connection) establishment the most unloaded $g$ grid resources that guarantee the minimum combined metric value. Metric can be representative of QoS parameters (e.g., 


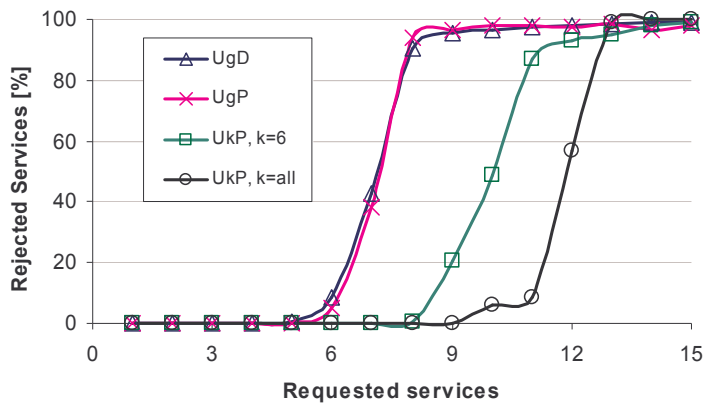

Fig. 1: Percentage of rejected services

communication delay) or, as considered in this study, of the amount of utilized network resources (i.e., overall bandwidth).

Simulation results

The UgD, UgP and UgPk schemes have been evaluated through simulations considering the NSF transparent network topology made of $N=14$ nodes and $W=8$ wavelengths per link. One grid resource per node is considered (i.e., $G=N$ ). A grid service requiring a full mesh of lightpaths between $g=4$ grid resources has been considered (i.e., $p=6$ bidirectional lightpaths per service). Services are sequentially established and never torn down. Grid resource load has initial null value and it is incremented by one upon service establishment. Service rejection occurs if the network resources are insufficient to guarantee all the required $p$ lightpath establishments. Fig. 1 shows the percentage of service rejection for the considered schemes. Results show that no advantages can be achieved by the concurrent computation of $p=6$ lightpaths performed by $\mathrm{UgP}$ with respect to the distributed set up performed by UgD (which exploits all the available GMPLS features like label set, acceptable label set, crankback). However, results show that significant reduction of service rejection is achieved by UgPk already with $k=g+2=6$, which requires $c=15$ combinations of $p=6$ computations. Fig. 1 also shows the optimal result obtained with $k=N=14$, which however may introduce scalability issues due to the high number of required path computations $(c=1001)$.

\section{Experimental results}

The considered schemes have been evaluated on the network testbed shown in Fig. 2. Among the $N=7$ nodes, the two core nodes are transparent OXCs while the five edge routers are connected to equally unloaded grid resources (i.e., $G=5$ ). Link capacity in terms of wavelengths (from w0 to w3) is depicted in Fig. 2. One grid service $s_{i}$ is considered requiring full mesh of bidirectional lightpaths among $g=4$ grid resources. If the UgD scheme is applied a $97.7 \%$ service rejection is experienced. Two conditions determine the service rejection. First of all, if the result of the random selection includes router $\mathrm{R} 5$, the service is rejected due to the lack of available wavelengths on link R5-OXC1. Moreover, even if the

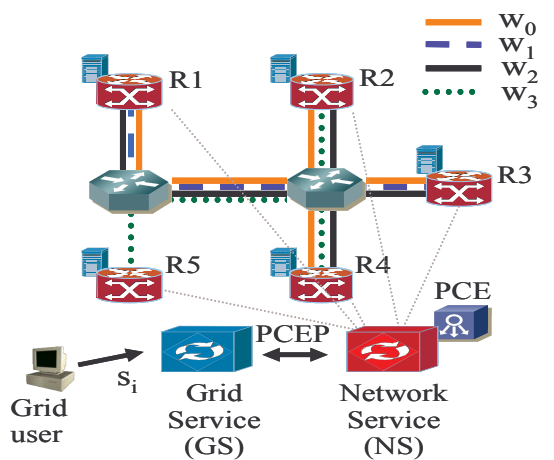

Fig. 2: Experimental setup

random selection excludes router R5, the service may also be rejected because of lack of network resources. This occurs if the distributed lightpath establishment does not allocate wavelength $\mathrm{w} 1$ for lightpath R1-R3 and wavelength w3 for lightpath R2$\mathrm{R} 4$. By adopting the UgP scheme, the latter critical condition is avoided thanks to the concurrent path computation performed by the PCE which identifies for each lightpath the proper available wavelength to use. However, the former critical condition related to the random choice of router R5 persists. With the adoption of the UgP scheme, service rejection is reduced to $80 \%$.By applying the UgPk scheme with $k=5$, the service is successfully established since both critical conditions disappear. The $\mathrm{c}=5$ combinations of $p=6$ lightpath computations allow to exclude router R5 and to allocate the wavelengths that avoid service rejection.

The adopted PCE implementation is derived from [4]. The PCE takes about $t_{c}=15 \mathrm{~ms}$ to compute each combination of $p=6$ lightpaths. Communication between Grid Service and Network Service running PCE is based on the current version of the PCE Protocol (PCEP) PCReq and PCRep messages. Lightpaths are setup by using RSVP-TE implementation for bi-directional lightpaths in [2]. The overall $p=6$ lightpaths activation is completed only after $t_{\text {tot }}=6 \mathrm{~s}$ and mainly determined by the time required to configure and activate node settings.

\section{Conclusions}

In this study a grid networking scheme exploiting the currently defined PCE architecture is proposed. The scheme allows a grid service to select from a large set of grid resources those that minimize the network resource utilization. Results show that the proposed scheme allows to significantly increase the amount of established grid services without compromising the service set up time.

Acknowledgements: This work has been partially supported by BONE project. We would like to thank W. Ren for the technical support in the experiment.

\section{References}

[1] L. Valcarenghi et al., Comm. Mag., Mar. 2006.

[2] S. Xu, et al, iPOP 07 Conf, June 07

[3] T. Tsuritani, NFOEC 08, Feb 08

[4] F.Cugini et al, OFC2007, March 2007 